\title{
Progress and Status of e-MERLIN
}

\author{
Ralph Spencer \\ Jodrell Bank Centre for Astrophysics, The University of Manchestr \\ Oxford Rd, Manchester, UK \\ E-mail: ralph.spencer@manchester.ac.uk
}

The upgraded MERLIN array of telescopes (e-MERLIN), is nearing completion with operations expected in early 2010. The correlator, being built by the Dominion Radio Astronomy Observatory of the Canadian NRC-CNRC is crucial to the performance of the instrument. Station boards and baseline (correlator) boards have been delivered. Signal paths have been tested - with an autocorrelation spectrum being obtained in April, and fringes shortly after. I will discuss some of the design features, in particular the data links, the phase cohering system and the interface to VLBI. The results of a successful call for Legacy observing proposals will be summarised, and finallly a report on the current progress and future plans will be given. e-MERLIN will have a major impact in high resolution radioastronomy, in fields from star formation and through all stages of stellar evolution, to the formation of galaxies at high redshift. This paper is presented on behalf of the e-MERLIN team at Jodrell Bank Observatory.

Science and Technology of Long Baseline Real-Time Interferometry: The 8th International eVLBI Workshop - EXPReSO9

Madrid, Spain

June 22-26, 2009 


\section{Introduction}

The e-MERLIN project [1] is a major upgrade to the MERLIN array run by the University of Manchester as a National Facility for the UK's Science and Technology Facilities Council. New low-noise receivers improve the system performance, however the main improvement in sensitivity to continuum sources comes from a huge increase in bandwidth over that available in MERLIN. The increase from $14 \mathrm{MHz}$ per polarisation to $2 \mathrm{GHz}$ is made possible through the use of a purpose-built optical-fibre network connecting the 7 MERLIN telescopes to the new correlator at Jodrell Bank Observatory. Including he Lovell 76-m telescope in the array results in a factor of $\sim 30$ improvement in senstivity at $5 \mathrm{GHz}$.

e-MERLIN has a unique combination of 50-150 mas resolution and $\mu \mathrm{Jy}$ sensitivity crucial to resolve AU-scale star-formation processes in our Galaxy and kpc-scale regions at $\mathrm{z} \sim 1$. Figure 1 shows how e-MERLIN compares with other instruments, and shows how it fills the gap bewteen the EVLA and eVLBI.

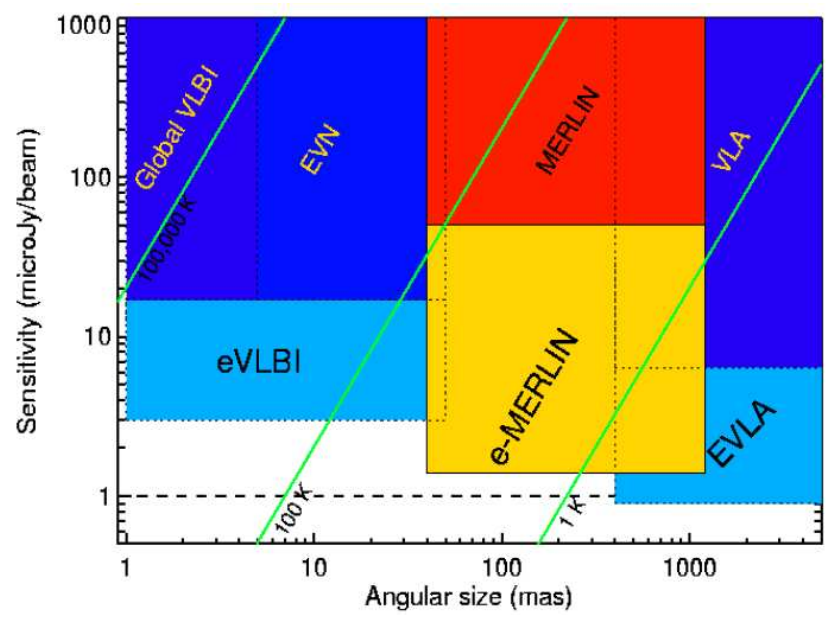

Figure 1 The sensitivity and resoution of e-MERLIN compared with other instruments.

The following sections discuss the expected performance, the receiver system, data links. the interface to e-VLBI and Legacy progammes. Finally the current status and future plans are described.

\section{Expected performance}

The baselines in e-MERLIN extend to $217 \mathrm{~km}$, and hence give 150, 40 and 10 mas resolution at $\mathrm{L}[20 \mathrm{~cm}], \mathrm{C}[6 \mathrm{~cm}]$, and $\mathrm{K}[1.3 \mathrm{~cm}]$ band respectively. The wide bandwidth of eMERLIN and new receivers will result in C-band sensitivities of $\sim 30 \mu \mathrm{Jy}$ r.m.s. in 1 minute, $\sim 2 \mu \mathrm{Jy}$ in typical runs and sub $\mu \mathrm{Jy}$ in deep fields. Spectral imaging over 1.3-1.7 and 5-7 GHz gives improved u,v coverage (figure 2 ) and the new correlator will allow wide field imaging out to the HPBW of a $25-\mathrm{m}$ telescope ( 9 arcmin at $\mathrm{C}$ band and 30 arcmin at $\mathrm{L}$ band), though the missing short spacings in the coverage will result in lower fidelity for extended source components, as is currently the case for MERLIN. The correlator is built on the WIDAR (WIdeband Digital ARchitecture) principle [2] where the incoming digital signal is split into 
16x128 MHz bands before further processing. The design makes extensive use of field programmmable gate arrays (FPGAs) and is very flexible in functionality.

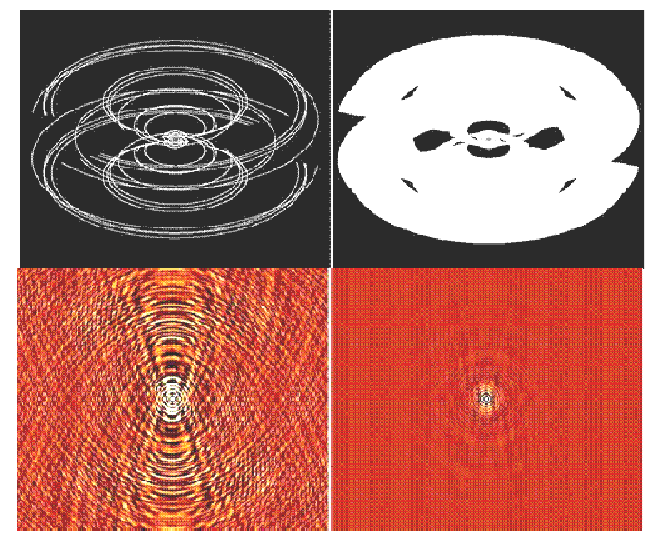

Figure 2 Aperture plane (u,v) coverage (upper) and point spread function (lower) for MERLIN (left) and e-MERLIN (right) at $\mathrm{C}$ band with 2 $\mathrm{GHz}$ total bandwidth.

In spectroscopic mode it has 16 placeable sub-bands each with more than 512 channels per sub-band per polarisation. Re-circulation will give much higher spectral resolution with up to 131072 channels per sub-band per polarisation [1]. The number of channels, polarisation, and bandwidths can be mixed and traded up to the maximum capacity of the correlator.The use of GPS meaurements of troposphereic delay will improve the astrometric performance of the array, with the goal of $<1$ mas with respect to the ICRF.

\section{Receiver System and Links}

The provision of wide bandwidth (e.g. a $4-8 \mathrm{GHz}$ receiver at $\mathrm{C}$ band) required a entirely new design for MERLIN's receiver systems. New intermediate frequency (IF) and local oscillator systems have been designed and are being produced. These require the signals to be up converted before amplification and filtering before being down converted to the $2-4 \mathrm{GHz} \mathrm{IF}$ suitable for input to the samplers.

Signals are digitised at the telescope, requiring extensive shielding to avoid locally induced RFI. Each $2 \mathrm{GHz}$ wide IF band (in 2 polarisations) is digitised to 3 bit precision, resulting in $24 \mathrm{Gbps}$, becoming $30 \mathrm{Gbps}$ after encoding and formatting. Three $10 \mathrm{Gbps}$ optical channels are used to transmit the data in parallel, where each electro-absorption laser (EML) is modulated in non return to zero (NRZ) format. L-band signals are digitised at 1 Gsamples per second to cover the $512 \mathrm{MHz}$ bandwidth using 8 bits, producing a total data rate of $16 \mathrm{Gbps}$ and 20 Gbps after encoding etc., thus effectively only two of the optical channels are used here. The digital systems, designed by NRAO in collabration with JBO are very similar in design to those used for ALMA and the EVLA. The input to the correlator is via the same station board design as the EVLA, where 3 fibres each carrying $10 \mathrm{Gbps}$ go into the station board where the light is photodetected and the data deformatted before being further processed.

The digital data rates used by e-MERLIN are far in excess of the currently (or even in the next few years) available capacity of the academic links in the UK. We initially investigated managed bandwidth solutions when designing the link system, but this proved far too expensive. Instead a dark fibre solution was achieved, using leased dark fibre where possible 
and new dig (the "last mile problem") to the telescopes. In total there is $670 \mathrm{~km}$ of fibre with $\sim 100 \mathrm{~km}$ of new build (figure 3). The new build was done by Fujitsu Telecommunications Europe Limited [3], with the previously intalled fibre mostly provided by Global Crossing [4].

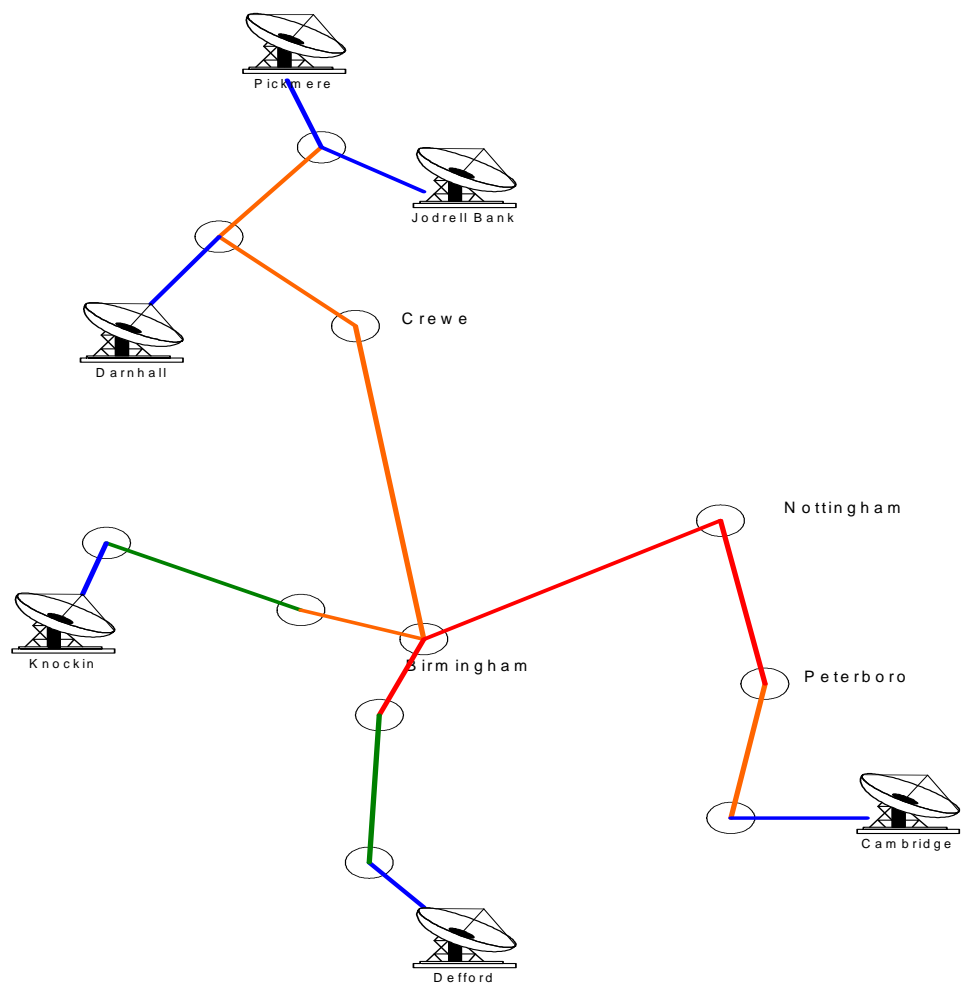

Figure 3 Schematic of the eMERLIN optical data link system. The red shows previously installed leased fibre, the blue the new dig connections to the MERLIN telescopes. The optical design was done by staff at JBO, all fibres are leased with maintenance being performed by the commercial providers

Data from the more distant telescopes (Cambridge, Defford and Knockin) are wavelength division multplexed at Birmingham, resulting in 9 channels on a fibre. The data flow is oneway, apart from that for control and monitoring which is on a separate telecommunications system.

MERLIN currently achieves coherence by the use of a reference radio frequency signal (at $1.5 \mathrm{GHz}$ ) transmitted (via repeaters) to each telescope site from JBO where the transmitter is locked to the Hydrogen Maser frequency standard. The signal is pulse coded, and re-transmitted back to JBO, thereby producing a go-and-return system. The signal is used to lock a high quality crystal oscillator at the telescope with a long $(>10 \mathrm{sec})$ time constant and so short timescale variations in the path length are averaged out. The diference in phase between go and return signals is used on-line in the correlator to correct the astronomical phases for slow path length changes in the reference link (anounting to peak to peak changes of several nsec in a day). This arrangement has worked very well, achieving pico-second stability, but has to be superseded, and so we have investigated the use of fibre optics using the installed fibre instead of the free space system. A number of laboratory and field tests show that use of the phase transfer system in modulating light on fibre instead of driving antennas works well [5]. Figure 4 illustrates the comparative behaviour of free space and fibre links showing the superior stability of a fibre system even before correction using the go and return signal. 


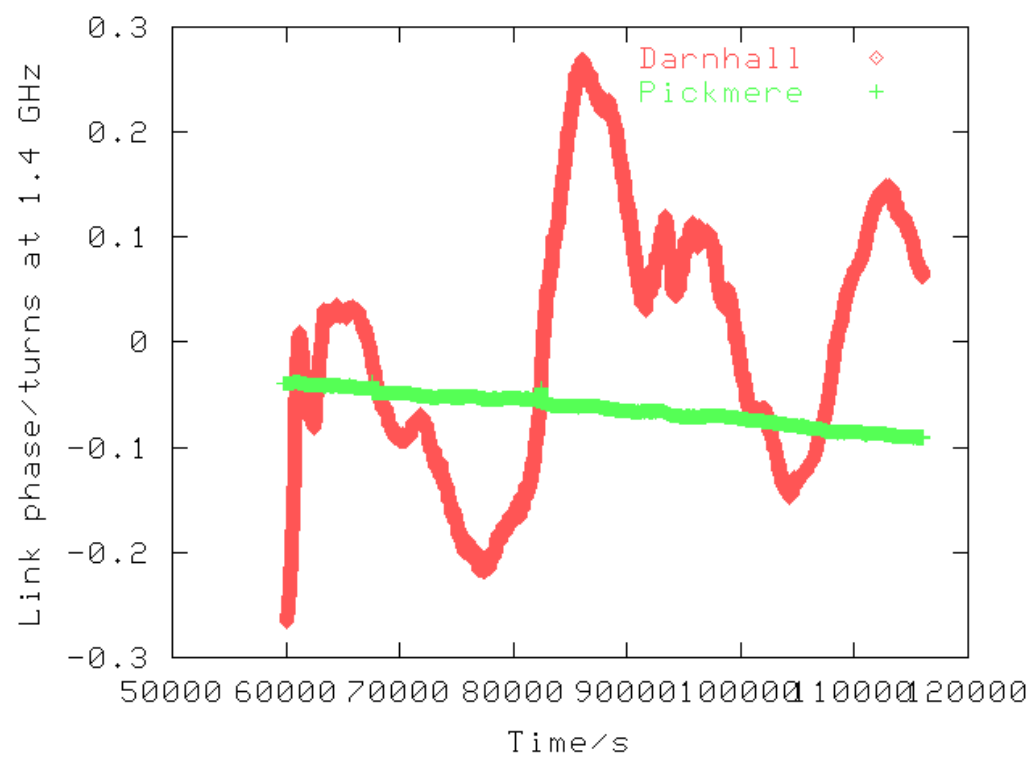

Figure 4 Graph showing the go and return link phase (in turns at $1.5 \mathrm{GHz}$ ) for JBO to Darnhall using the MERLIN free space antenna based system (red), and JBO to Pickmere using optical fibres (green), over $\sim 1$ day. The two path lengths are within a factor of 2 and were measured simultaneously.

\section{Connection of e-MERLIN to e-VLBI}

The incorporation of multiple MERLIN telescopes into VLBI has scientific benefit due to tncreased sensitivity and the addition of valuable short spacings. There has been consistent demand for this facility from the VLBI astronomical community for several years. The use of the new digital links, though enabling very high bandwidth, presents an interfacing problem for VLBI since data formats are very different. Work packages in the EXPReS project were designed to overcome this. The work packages were 1) to allow up to 4 telescopes at $1 \mathrm{Gbps}$ each to be connected to JIVE (in SA1) and 2) to connect data from Onsala into the e-MERLIN correlator at 4 Gbps (in FABRIC/JRA1), allowing tests at higher data rates than possible with the JIVE correlator. A so called VSI FPGA chip on the station board is designated for providing a high data rate parallel digital interface - where signals can be routed either in or out to the correlator. The interface to the IP network (for e-VLBI in particular) has been achieved by the use of the University of Berkeley's CASPER groups internet break-out boards (iBOB) [6]. These FPGA boards have been also developed by us as network testers, where high speed (10 Gbps) can be tested over a range of data rates using UDP packets and errors counted. Tests to Onsala have shown that the link can transmit continously at 4 Gbps with no packet loss (see talk by Richard Hughes-Jones at this conference). Test data from Onsala has been passed into the correlator, and autocorrelation spectra obtained.

Firmware for passing data from e-MERLIN telescopes out to the JIVE correlator, which rechannelises and removes the delay corrections, has been developed, though further tests are needed. We do not expect to get full connectivity until after further e-MERLIN telescopes have been brought into operation. In the meantime the existing analogue links are being used for eVLBI observations using data rates of $128 \mathrm{Mbps}$ for the remote telescopes and up to 1024 Mbps for the local Mk2 or Lovell telescopes. 


\section{The Legacy Programme}

e-MERLIN will be used both for standard rsponse mode observations via the usual twice yearly proposal system and for large scale 'Legacy' projects. A call for large high scientific impact projects, aimed at reaching a wider community than that typical for MERLIN and VLBI, resulted in 15 proposals submitted from 325 scientists from 119 institutes, clearly indicating a wide community interest. The proposals were reviewed by external referees and a Legacy Steering Group, chaired by Prof. R. Ivison. Observing time was well over-subscribed with 5000 hours allocated to 11 successful projects, covering topics from the formation of planets to the formation of galaxies in the early universe. Around $~ 50 \%$ of e-MERLIN time in the first 5 semesters will be allocated to the Legacy progammes. The breadth of the proposals clearly demonstrates the versatility of e-MERLIN [7], and the whole process was an important demonstration of community interest and potential scientific output.

\section{Current Status and Future Plans}

First light through digital lnks and the e-MERLIN correlator occured in early April 2009, followed by an autocorrelation spectrum of methanol being obtained on April 13th. The first fringes between the Mk2 and Tabley telescopes were obtained on April 24th. Since then fringes have been stabilised, with correct delay tracking. At the moment we have 4 station boards and 1 baseline board. The rest of the correlator boards are expected to be delivered in the Autumn 2009. All receiver font ends have been completed, though further improvements to the $\mathrm{K}$ band receiver are scheduled. It is planned to incorporate further telescopes over the next few months, with initial test and science runs usin the full array in early 2010 .

\section{Acknowledgements}

Many thanks are due to the e-MERLIN and VLBI teams at JBO. Special thanks are due to Simon Garrington and Rob Beswick, for valuable discussion and provision of slides.EXPReS is funded by the European Commission (DG-INFSO), under the Sixth Framework Programme, Contract \#026642.

\section{References}

[1] e-MERLIN Web site http://www.e-merlin.ac.uk/, viewed 27/7/09.

[2] WIDAR correlator https://mitr.drao.nrc.ca/widar/index.shtml viewed 27/7/09

[3] Fujitsu Press Release http://www.fujitsu.com/uk/news/pr/ftel_20090514.html, viewed 30/07/09

[4] Global Crossing web site http://www.globalcrossing.com/ viewed 30/07/09

[5] McCool, R., et al. $40^{\text {th }}$ Precise Time and Time Interval meeting December 2008 http://www.pttimeeting.org/archivemeetings/ptti2008.html\#program viewed 4/8/09

[6] CASPER web site http://casper.berkeley.edu/wiki/IBOB viewed 31/7/09

[7] e-MERLIN Legacy Projects web site http://www.merlin.ac.uk/legacy/ viewed 31/7/09 